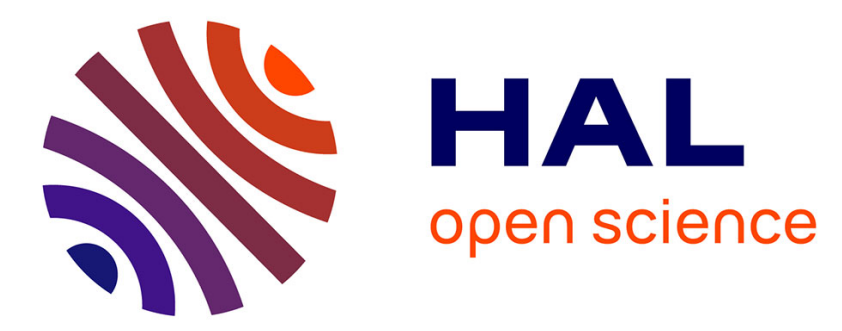

\title{
Innovation Ecosystems: A Collaborative Networks Perspective
}

\author{
Ricardo J. Rabelo, Peter Bernus, David Romero
}

\section{To cite this version:}

Ricardo J. Rabelo, Peter Bernus, David Romero. Innovation Ecosystems: A Collaborative Networks Perspective. 16th Working Conference on Virtual Enterprises (PROVE), Oct 2015, Albi, France. pp.323-336, 10.1007/978-3-319-24141-8_29 . hal-01437900

\section{HAL Id: hal-01437900 \\ https://hal.inria.fr/hal-01437900}

Submitted on 17 Jan 2017

HAL is a multi-disciplinary open access archive for the deposit and dissemination of scientific research documents, whether they are published or not. The documents may come from teaching and research institutions in France or abroad, or from public or private research centers.
L'archive ouverte pluridisciplinaire HAL, est destinée au dépôt et à la diffusion de documents scientifiques de niveau recherche, publiés ou non, émanant des établissements d'enseignement et de recherche français ou étrangers, des laboratoires publics ou privés. 


\title{
Innovation Ecosystems: A Collaborative Networks Perspective
}

\author{
Ricardo J. Rabelo ${ }^{1}$, Peter Bernus ${ }^{2}$, David Romero ${ }^{3,2}$ \\ ${ }^{1}$ Federal University of Santa Catarina, Brazil \\ ${ }^{2}$ Griffith University, Australia \\ ${ }^{3}$ Tecnológico de Monterrey, Mexico \\ ricardo.rabelo@ufsc.br, p.bernus@griffith.edu.au, david.romero.diaz@gmail.com
}

\begin{abstract}
Innovation ecosystems (IE) have increasingly gaining importance due to their potential to leverage regional development. In a previous research, authors have translated into processes how current IEs have been built or emerged. This process-based model can be used for building new or support the analysis of existing IEs. In order to evaluate the completeness of this model, this paper presents its mapping against the ARCON reference model considering that an IE can be seen as special type of Collaborative Network. Given the particularities of IEs, this paper also provides some elements of reflection that may be taken into account in future ARCON evolutions.
\end{abstract}

Keywords: Innovation Ecosystems, Collaborative Networks, Reference Model.

\section{Introduction}

Innovation ecosystems (IE) have been nowadays considered as the most prominent driver to be built up and nourished to reap the benefits of innovation. This reflects a paradigm shift, whereupon innovation is becoming a centrepiece of a socio-economic development model for cities and regions [1]. An IE can be defined as an environment and economic development and diffusion model formed by an ecology of actors whose goal is to create, store and transfer knowledge, skills and artefacts which define new technologies, enable technology development and innovation, made up of interconnected institutional entities (e.g. industry, academia and government) participating in the IE, bound together by social interactions and culture [2] [3].

Building an IE is a more complex task compared to other environments that are typically less open and more controlled, like incubators, technology and science parks, innovation habitats and centres, or virtual organisation breeding environments (VBE) [5] [6]. When seen as a whole, this organic task comprises different and independent but interrelated activities that must be performed carefully considering different tangible and intangible matters. Such activities (being implicit or deliberate, emergent or planned, static or evolving, loosely or tightly managed) span the IE's life through all stages of its evolution [7].

An analysis of literature reveals no consensus about the required lifecycle phases, involved processes, their recommended sequence or stages of evolution; nor is literature definitive about the actors and enabling elements most likely to play the major roles in building an IE [8]. Most of the consulted works focus on some specific phases; e.g., how to qualify different actors; how to analyse a region to better identify its business vocation; how to conduct innovation processes inside the IE; etc. This 
lack is also evident in research projects that deal with enabling innovation ${ }^{1}$.

This paper extends and complements previous work by the authors [7], which identified and represented processes that were involved in building and sustaining existing IEs. However, in order to both serve as a guide for future IE building, and for refinements of current IEs, it is important to check how complete the devised model is. Therefore, this paper is not about innovation models themselves. Adopting as the initial hypothesis that IEs can be seen as a Collaborative Network (CN) [10], authors organised and mapped the identified processes against ARCON (A Reference model and Modelling framework for Collaborative Networks) [11], which is seen as the most relevant model for CNs. One of the advantages of analysing IEs from the CN perspective is the possibility to apply the huge bunch of knowledge on collaborationbased networks when investigating the several issues of IEs.

The remaining part of the paper is organized as follows. Section 2 summarizes the adopted research methodology. Section 3 identifies the differences and commonalities between IEs and CNs. Section 4 gives an overview about the IE model. Section 5 maps this model against ARCON. Section 6 discusses this mapping, highlighting at which extent ARCON can be used to model IEs. Section 7 presents the conclusions.

\section{Basic Research Methodology and Underlying Concepts}

This qualitative research work was carried out based on conceptual analytical method according to Järvinen's taxonomy of research methods [12]. The work includes three research actions to achieve its goals: (A) the study of IEs as a type of $\mathrm{CN}$; (B) the mapping of the devised process model against ARCON and the identification of possible gaps in the former; and (C) the identification of IE specific details that could be incorporated into ARCON in order to help its users when specifically applying it for the creation of future IEs.

For (A) a systematic literature review was performed, looking at definitions of IEs and conceptual foundations of CN-like networks. For (B) ARCON was studied and compared against the IE lifecycle process model. Authors highlight the diversity of terminologies used when describing/characterising what an IE is. For (C) the commonalities and particularities of IEs against ARCON were identified.

In terms of the present treatment, a combination of terminology established in the systems engineering community ISO 15288 [13] and enterprise engineering community ISO 15704 [14] was used. Thus, by a process $(P)$, we mean a collection of inter-related activities $\left(a_{i}\right)$ that transform physical goods and/or information (the input) into output by performing a value adding function $(F)$. Activities in a process are performed by resources $\left(r_{i}\right)$, which in turn are entities considered to be systems capable of performing a set of (more elementary) functions $\left(f_{j, k}\right)$.

\footnotetext{
${ }^{1}$ e.g. The European projects BIVEE, ComVantage, IMAGINE, CoVES, Laboranova, PLENT, GloNet and SmartNets [9] have tackled innovation with different models, platforms and scopes, basically supporting the collaborative development of products and related services mainly for the manufacturing sector. They essentially focus on the "ecosystem" operation stage and not on how to build it.
} 


\section{Innovation Ecosystems and Collaborative Networks}

In order to better understand the nature of these 'ecosystems' and if they can be considered as a type-of $\mathrm{CN}$, we looked at literature for definitions of an IE (see below) to compare these against the definition of CNs:

- "An environment with economic agents and economic relations as well as the non-economic parts, such as technology, institutions, sociological interactions and the culture" [3]

- "Networks that provide mechanisms for goal-focused creation of new goods and services tailored to rapidly evolving market needs, with multiple, autonomous and independent institutions and dispersed individuals for parallel innovation" [15]

- "Independent factors working together to enable entrepreneurs and allow innovation to occur in a sustained way in a particular location" [16]

- "An environment and economic model formed by actors whose goal is to enable technology development and innovation", made up of institutional entities participating in the ecosystem, bound together by social interactions and culture [2] [3] (paraphrased)

- "An environment that aligns independent actors, regulations and supporting elements to leverage actors playing their roles in an organised and collaborative way towards developing innovations" [17]

- "An open, dynamic, sustainable and evolving networked business environment, which catalyses and drives the transformation of ideas into valuable outcomes under varied business models, supported by capital and by heterogeneous actors' knowledge and infrastructures, constrained by policies, regulations, governance and culture" [7]

- "The inter-organisational, political, economic, environmental, and technological systems of innovation through which a milieu conducive to business growth is catalysed, sustained and supported" [...] "It is characterised by a continual realignment of synergistic relationships that promote harmonious growth of the system in agile responsiveness to changing internal and external forces" [18].

Elements of an IE include [2] [4] [8] [15] [19]: Actors (government, universities, industry, supporting institutions and specialised people, entrepreneurs, financial system, customers and civil society, and their [social and economic] relationship, playing various roles throughout the IE's life); Capital (financial assets provided by some actors); Infrastructure (physical, technical conditions and general resources to support the IE and the innovation developments 'inside' of it); Regulations (laws and rules that frame the IE functioning and innovation environment); Knowledge (existing supporting theoretical foundations, tacit and explicit, formal, informal and specialised knowledge that are used, generated (and eventually organised and managed), made available, and learned along the innovation value chain); Ideas (intentional thoughts that trigger innovation actions and around which the whole IE works).

Three additional elements impact the way the IE operates [7]: Interface represents the channel to support the interaction between the IE's participants with external actors, considering their usually significant heterogeneity; Culture refers to the mindset of people and organisations combined to support and easy innovation initiatives and to solve related problems [4]; Architectural Principles refers to the way the IEs' 
elements are combined, orchestrated and the culture element is also reflected in them.

The IE's dynamics makes actors assume multiple, but not fixed or pre-defined roles, in the different stages and involved phases of IE life.

A Collaborative Network $(\mathrm{CN})$ is defined as [10] "a network constituted by a variety of entities (e.g. organisations and people) that are largely autonomous, geographically distributed, and heterogeneous in terms of their: operating environment, culture, social capital, and goals" [...] "CN focus on the structure, behaviour, and evolving dynamics of networks of autonomous entities that collaborate to better achieve common or compatible goals" [...] "interactions are supported by computer networks" [...] "CN collaboration derives from the shared belief that together the network members can achieve goals that would not be possible or would have a higher cost if attempted by them individually".

Based on these definitions and related literature [4] [15] [18] [20], we argue that an Innovation Ecosystem is a $\mathrm{CN}$ as it has all essential $\mathrm{CN}$ characteristics, namely: formed by autonomous, independent, distributed and heterogeneous actors [...] that behave, interact and collaborate with each other with different roles [...] in a sociotechnical network [...] within a fertile, spatial and evolving environment [...] to overcome individual capability limitations, maximise resource usage, and share risks and costs, [...] so as to better achieve common/compatible goals [...] regarding the different involved cultures [...] and intrinsic network dynamics. Although not explicitly mentioned in the above $\mathrm{CN}$ definition, trust is also a crucial issue in IEs.

As a matter of fact, it is not the name or borders of the network that turn it into a $\mathrm{CN}$, but rather what and how things happen inside of it.

There are many other dimensions to characterise a $\mathrm{CN}$, like if it is mono or multisectorial, long-term or grasp-driven, regionally or globally focused/placed, etc. However, when looking at more specific features of typical IEs, some major differences can be pointed out. The ARCON reference model identifies fourteen basic types of CN [11]. Two of these are the most similar to IEs: Business Ecosystem and Virtual organisation Breeding Environments (VBE) long-term alliances. An IE is a broader concept and is a more open and dynamically emerging environment than a Business Ecosystem (in the ARCON reference model characterised similarly to an industrial cluster). Original VBE concepts [21], its so-called 'second generation' [22] and inter-played CNs [23] still represent 'closed-world' type of alliances, although allowing multi-sectorial companies and inter-VBE collaboration to better support the creation of virtual enterprises/organisations.

It is important to highlight that it is not our goal here to verify if an IE is 'better' or not than e.g. a VBE. Instead, the point is that IEs seem to be a particular type of CN suitable to achieve certain strategic goals and objectives. The main similarities and differences between IEs and other CNs include:

- $\mathrm{CN}$ original definition stands for having computers networks as the means to support interactions among members. Although ICT can be very much variable in type and usage intensity, and regarding its current proliferation in the society and organisations, it is rather difficult to imagine IE's members interacting and doing their work without using ICT reasonably intensively, in the same way as other types of CNs.

- An IE is not always created as a methodologically planned and induced initiative of some actors. There are several cases (e.g. Silicon Valley) which have simply emerged as a result of a set of regional factors. In cases of more planned initiatives, its building is far from being linear or sequential. Its 
building phases are very much decoupled from one to another, and processes' phases and actors evolve at different pace and independently from the other ones.

- An IE typically embraces many kinds of actors, existing infrastructures and even other CNs. Because there is no physical or organisational border, IEs embrace universities, private R\&D labs, funding agencies and banks, etc., besides previously established industrial clusters, innovation habitats and VBEs. Therefore, IEs can be considered as a 'logical' environment on top of existing $\mathrm{CN}$ and other non-CNs alliances. IEs can also interplay with other ones.

- Actors can perform several different roles throughout the IE's life, having plenty of members' capabilities overlapping.

- Actors may be different in nature, internal processes, stages of evolution/maturity level, and value systems. Therefore, an IE can be seen as a heterogeneous system of systems. Although being independent entities, actors may perform actions related to sustaining the entire IE and not only to the operational actions related to various innovation initiatives, e.g., some actors help other actors to be created and evolve.

- The so-called 'minimum level of preparedness' that each actor should have to collaborate is much less formalised, controlled and homogenous than in other types of CNs. Although 'preparedness' can be used as one important criterion for partners selection or suggestion, practice in IEs shows that this is mostly resolved 'on the fly'.

- Joining and exiting of actors can be dynamic and even unnoticed. IE boundaries are intrinsically 'elastic'. This means having only general and less formal governance and performance management models: the IE manages itself in an organic manner rather than being managed by some central authority. Due to cultural factors and implicit social rules it is unlikely for a formal governance to strongly coerce members and system behaviour.

- IEs involve another level and nature of outcomes. Besides generating physical outcomes, less tangible or more abstract impacts are just as important. This requires the identification of adequate performance indicators aligned to the IEs' goals, but observable by all as a feedback mechanism.

- An IE is devoted to conduct, leverage and sustain innovation and to boost business and (real rather than virtual) enterprises creation;

- The creation of Virtual Enterprises/Organisations (VE/VO) can be seen as a possible consequence - and not as an ultimate purpose - of a given innovation initiative. There are four differences compared to 'classical' VE/VO: First, innovation can happen anytime in the IEs' phases and processes performed by actors involved in, creating multiple and simultaneous value chains. Second, an innovation initiative includes partners not having 'common' goals in the strict sense. Actors have their own intrinsic interests aligned to the type of 'system' they belong to. Therefore, when a $\mathrm{CN}$ is defined to 'achieve common or compatible goals', in the context of IEs perhaps a more appropriate formulation might be as 'support the achievement of mutually beneficial but independent/aligned goals'. Third, innovation outcomes not necessarily refer to final physical goods to be delivered or ready commercialisable ideas. Sometimes the goal is to test a concept or technology; intermediate results may 
be exploited in different ways and by another VOs; etc. Fourth, the VE/VO composition can vary depending on the innovation needs, the required path and the innovation model in use. A significant part of a VE/VO is created 'on the fly', rather than follows the classic, usually linear and coupled steps of opportunity identification $\because$ VO/VE characterisation and planning $\because:$ partner search and selection $::$ negotiation $::$ contracting $:$ launching VE/VO. Many other 'sub' VE/VOs can emerge as the result of such dynamics.

- The classical role of the so-called 'VO Coordinator' does not necessarily exist. Coordination is achieved through organic negotiations between stakeholders as defined by the needs of the business and exploitation plan. This kind of network may use multiple network brokers and orchestrators who are in charge of finding the most suitable partnerships as the innovation project goes on.

All these particularities make IEs extremely difficult to build and integrate (including at the ICT level); they are perhaps the most complex type of $\mathrm{CN}$ when considering those fourteen types identified in ARCON.

\section{The Innovation Ecosystem Model}

This section presents the lifecycle phases and processes involved in the building of an IE (Fig. 1), and were inspired by the description [21] of VBE evolution. The IE's stages of life are named according to ARCON [11]. In Figure 1 processes are presented in a condensed way as the details are not essential to achieve this paper's objective. A very detailed description of each process, and how they were identified and derived from the current body of knowledge on IEs, can be found in [7].

When discussing the processes involved in creating, operating, changing, etc. of a system (such as an IE or in general a collaborative network) it is customary to categorise these processes according to the level of abstraction at which they consider that system. This is done by defining (from abstract to concrete) lifecycle 'processes' that define the identity, develop the concept, specify the requirements, and design, build, operate, and decommission the system. These types of processes are called lifecycle phases, due to the fact that their instances are repeatedly executed, often in parallel, and there is considerable amount of feedback (constraining relationships) involved. In the context of IEs, lifecycle processes cannot be seen only from the classical engineering perspective, where processes are always deliberately performed. In the present organic context, processes are often related to social phenomena, which in turn are largely unpredictable, dynamic, unstructured, emergent, and are only up to some extent observable and manageable. 


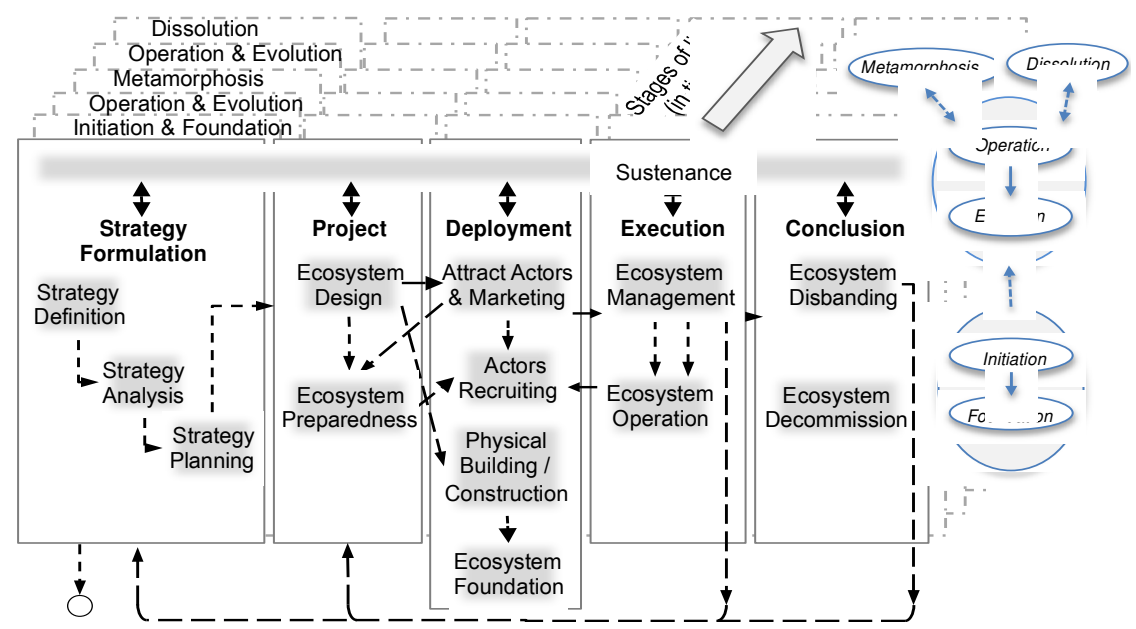

Fig. 1. Innovation Ecosystem: a) Lifecycle Phases and b) Stages of life

A system can evolve throughout its life in stages, and each stage can involve the execution of the same type of lifecycle processes many times over.

The evolution of a system may involve various kinds of change, like the joining of new members, replacement of parts of the system, creating new connections and relationships, learning, restructuring, developing new assets that enable previously impossible functions, etc., so a system evolves throughout its life, at the same time as it is operating. Part of this evolution includes the improvement of the system lifecycle processes themselves. Improvements include changes in maturity, emergent or induced growth, learning and gaining experience, making autonomous decisions within the IE. Improvement may also be due to external events, or feedback from internal processes, or the external environment that cause or enable processes to change.

Both the system as a whole and supporting subsystems have stages of life that together cover their respective lifespans, and each have a respective lifecycle, that consist of their specific (lifecycle) processes (types).

Each phase has processes (e.g. Project consists of ecosystem design and ecosystem preparation processes) and activities (the set of actions performed within each process). Process scopes are not always perfectly determined and the control and information flow can vary due to the intrinsic non-linearity of enacting an IE (represented as dashed lines in Fig.1).

These processes are continuously performed/instantiated throughout the stages of the IE life. The IEs is initially devised, prepared, set-up and launched (initiation \& foundation), it reaches operation and gradually evolves as it goes (operation \& evolution), and may need deep changes in its identity (metamorphosis), or can even reach closure (dissolution). Note that we define 'metamorphosis' as substantial change in some of the identity attributes of the IE, but still the result of such deep transformation is rooted in the IE's earlier existence. If this were not so, then we would talk about 'dissolution'.

What follows is a description of the lifecycle phases. Note that the name 'phase' of 'lifecycle phase' suggests that these 'phases' consider the IE on different levels of 
abstraction. If we go from left to right in Figure 1, these processes reveal more and more concrete detail, and - as the phases of the Moon - they repeat by being instantiated during the stages of the IE's life, as well as use information feedback from previous instantiations of any of the phases through evolving cycles.

- Strategy Formulation phase: take strategic decision of creating a new or reinforcing an existing IE. This phase has three main processes: Strategy Definition (re)identifies the IE's mission, vision, values, performs feasibility analysis, and strategic goals. Strategy Planning defines actions plans and milestones, critical success factors and key indicators for the IE to be built or already running, actions to ensure preparedness of actors, defines mandates and overall plans, dedicated IE-building or transformation programs, and projects. Strategy Analysis refers to the variety of strategic analyses informing the evaluation and feedback of the outcomes of the two other processes.

- Project phase: design and take all steps to prepare the underlying conditions for building or transforming the IE. It has two main processes: Ecosystem Design defines the IE's 'architecture', its components, types of actors, roles and relationships, infrastructure requirements, governance model, operating and business models, bylaws, code of ethics, incentives and mechanisms to attract actors. Ecosystem Preparedness defines a plan of actions related to preparing involved actors, infrastructures, laws and regulations to cope with the IE's requirements, mid- and low level specifications, along the future stages of evolution.

- Deployment phase: formally establish the designed IE, transform specifications into infrastructures and populate with real actors. This has four main processes: Actors' Attraction \& Marketing designs and executes actions to publicise the IE to attract qualified actors. Actors' recruiting aims to attracting participants according to preparedness directives and rules. Physical building makes available suitable facilities to support the diverse types of actions required throughout an innovation's lifecycle, following the requirements and guidelines indicated in the design sub-process. Ecosystem foundation refers to the official organisational foundation of the IE, when pertinent. Depending on the deployment model and taxation laws as well as legal incentive mechanisms, this can involve a legal or more formal establishment of the IE, or in the other extreme case this may simply take the form of an 'announcement'.

- Execution phase: is the set of processes involved in the operation of the entire IE. It has two main processes: Ecosystem operation consists of the activities involved in creating and bringing to successful conclusion various innovation initiatives, as carried out 'inside' the IE. Ecosystem Management includes management activities of the IE, and can cover two levels: the strategic management of the IE itself (identifying opportunities, threats, issues, etc., and initiating other relevant lifecycle processes as above); the tactical and operational management of the IE. This process involves dealing with human resources, financial, organisational, technological, governance issues, and is likely to be a distributed collaborative set of activities, rather than being concentrated into a management role performed by any one particular organisational or individual actor.

- Conclusion phase: this phase is basically responsible for handling issues that deeply impact the continuation of the IE's life. This phase has two main processes: Ecosystem Decommission refers to handling the coming and going of 
actors within the IE along its life cycle. Ecosystem Disbanding refers to a gradual exiting of actors from the IE business environment due to e.g. strategic changes and general disagreements.

- Sustenance phase: this phase is responsible for handling the future evolution and viability of the IE, managing the IE's life cycle. This phase has one main process, but which crosses, impacts and receives feedbacks from all the other sub-processes. Ecosystem sustainability corresponds to tactical and strategic management levels that all phases have when performing their actions.

\section{Mapping ARCON and Innovation Ecosystems}

ARCON is a reference model and modelling framework directed to collaborative networks. It intends to be used as a basis for derivation of other specific models for particular cases in various types of $\mathrm{CNs}$, helping to understand the involved entities and significant relationships among them [11].

For the purpose of modelling all features of $\mathrm{CN}$ components, ARCON considers three perspectives [11]. The first one (ARCON lifecycle) addresses the timing of CN's life stages: creation, operation, evolution, metamorphosis and dissolution. The second perspective ('environment characteristics') focuses on capturing the CN's general features. This perspective in turn includes two so-called subspaces: The endogenous elements sub-space embraces the internal $\mathrm{CN}$ elements' characteristics and are classified into four dimensions: structural $(S)$, componential $(C)$, functional $(F)$ and behavioural (B). The exogenous interactions sub-space handles the logical surrounding of $\mathrm{CNs}$ and are also classified into four dimensions: market $(M)$, support $(S)$, societal $(S)$ and constituency $(C)$. The third perceptive ('modelling intent') refers to the different goals one may have when modelling a $\mathrm{CN}$, addressing the three possible intents: general representation, specific modelling, and detailed specification/implementation modelling. Figure 2 presents a very general 'instantiation' of ARCON.

This instantiation has the intent of representing the phases (groups of processes) involved in creating or transforming $\mathrm{CNs}$ of the type 'innovation ecosystem' at the specific modelling level. 


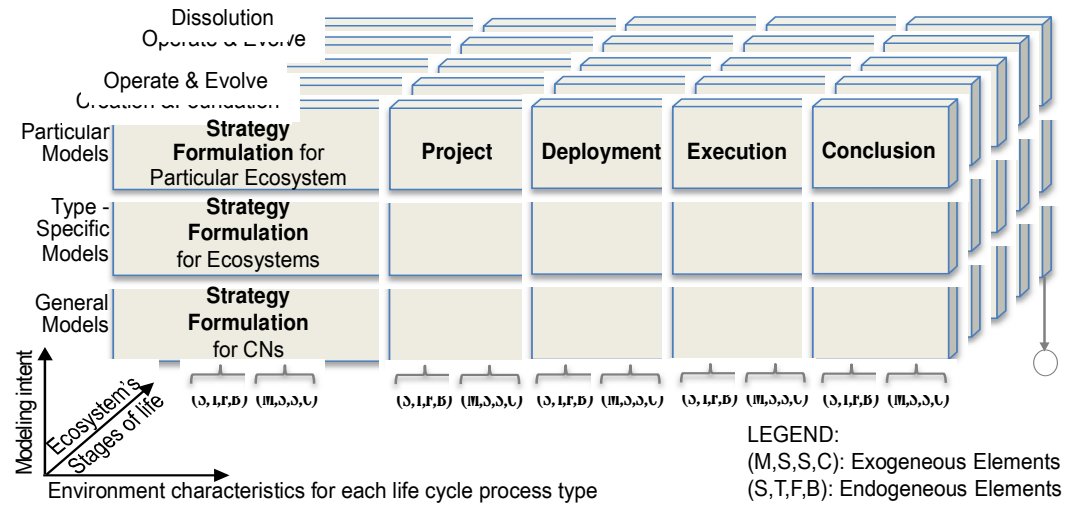

Fig. 2. The Innovation Ecosystem lifecycle phases framed by the ARCON model

Table 1 shows a small and simplified excerpt of the IE's modelling regarding the ARCON's environment characteristics perspective for one of the processes within the Project phase. As it will be discussed and is illustrated in Fig. 2, given the decoupled way of the IE's life cycle processes, these processes present a high level of independence from each other in terms of evolution (life cycle perspective). The instantiation of the endogenous elements and exogenous interactions can vary in the same process depending on the evolution stage of the IE, existing level of technical maturity, managerial experience, basic general conditions, and eventual inter-dependence with other processes.

Table 1. Example: Ecosystem Preparedness process - Environment Characteristics perspective

\begin{tabular}{|c|c|c|c|}
\hline \multicolumn{4}{|c|}{ Innovation Ecosystem Endogenous Elements sub-space } \\
\hline Structural & Componential & Functional & Behavioural \\
\hline $\begin{array}{l}\text { Active Entities } \\
\text { Actors: universities, } \ldots \\
\text { Roles: member, service } \\
\text { provider, coacher, ... } \\
\text { Concepts } \\
\text { Relationships: funding, ... } \\
\ldots\end{array}$ & $\begin{array}{l}\text { Passive Entities } \\
\text { Human Resources: } \\
\text { recruiter, orchestrator, } \ldots \\
\text { Knowledge Resources: } \\
\text { members profile \& } \\
\text { competency info, ... } \\
\ldots\end{array}$ & $\begin{array}{l}\text { Actions } \\
\text { Processes: eco membership } \\
\text { mgmt., strategic mgmt., ... } \\
\text { Concepts } \\
\text { Methodologies: training } \\
\text { method, coaching method,.. }\end{array}$ & $\begin{array}{l}\frac{\text { Concepts }}{\text { Behaviour: business }} \\
\text { culture, governance } \\
\text { principles, } \ldots \\
\text { Contracts \& Agreements: } \\
\ldots \\
\text { Incentives \& Sanctions: }\end{array}$ \\
\hline \multicolumn{4}{|c|}{ Innovation Ecosystem Exogenous Interactions sub-space } \\
\hline Market & Support & Societal & Constituency \\
\hline $\begin{array}{l}\text { Mission Statement: Eco } \\
\text { mission, vision, goals, ... } \\
\text { Marketing Strategies: ... } \\
\text { Market Interactions: } \\
\text { strategic customers, new } \\
\text { members, ... }\end{array}$ & $\begin{array}{l}\text { Network Social Nature: } \\
\text { profit-oriented eco, ... } \\
\text { Interaction Parties } \\
\text { Support Entities: } \\
\text { certification entities, ... }\end{array}$ & $\begin{array}{l}\text { Network Legal Identity: } \\
\text { Legal Name: "Eco Great" } \\
\text { Values \& Principles: free } \\
\text { commerce, free competition, } \\
\text {... }\end{array}$ & $\begin{array}{l}\text { Network Identity } \\
\text { Attracting Factors: } \\
\text { attracting \& recruiting } \\
\text { strategies, } \\
\text { Rules of Adhesion: ... }\end{array}$ \\
\hline
\end{tabular}

\section{Discussion}

Having in mind the differences between IEs and other CNs (see Section 3), it could be observed that the usual way of using ARCON as a process-based methodology seems not applicable at all here, because lifecycle processes are normally performed in parallel in a decoupled way, and not sequentially, refining the outcome in an evolutionary manner throughout the stages of the IE's life. 
Although decoupled, activities of these processes may be circularly dependent on one another. This can be easily understood by differentiating between activity as in process (type) definition (meaning 'activity type') and activity as in process instance (meaning 'activity instance'). This feedback in turn can be affected by the different pace and implementation success of each process' activities and policies. This means that an IE does not evolve linearly and harmoniously.

Therefore, modelling innovation systems should perhaps be done looking at each individual process level and not at once as if it was a "monolithic" block. This suggests a very high level of complexity - perhaps not being truly and tightly manageable - as each "step" of every single evolving stage is different among the phases/process as the processes evolve in a non-synchronised and non-linear way. A possible approach for this is to see the IE's processes from the fractal point of view, where each process might be independently (although not in an isolated manner), modelled as the image of the whole.

Processes' activities need specific and proper instantiations for the given IE instance, with consideration of the maturity levels of technical and management capabilities, local conditions, culture, planned goals, and the required and available investments. Actors' involvement in each process in terms of intensity of participation and roles can also vary due to emergent leaderships. In the case of the operation processes (the phase where innovation projects are usually performed), the derivation of particular models is probably only feasible if done by the people involved, using a library of elementary process definitions, such as described in Malone's Process Handbook [24]. This is because every single innovation project is carried out differently, in terms of partners, innovation model, (eventual) supporting ICT tools, governance model (if any), planned outcomes, IPR and transfer mechanisms, etc. In other words, there might be innumerable possible instantiations. Therefore, given the intrinsic "elasticity" and dynamics of IEs, it is an open point how feasible it is to derive a complete instance of a particular model, even via ARCON.

Other issues have to be tackled from wider views, as business models for example. Considering its role in terms of regional development, business models for IEs can be as many and should be handled at regional level, at the IE (as an entity) level, at the processes level, and at the individual innovation's level. Less tangible issues, like culture, are extremely critical in IEs. They are difficult to be understood and hence modelled. In this aspect, ARCON leaves to the "derivers" the option of using whatever tools and approaches when intending to derive particular instances of CNs; e.g. via soft modelling methods [11].

Another aspect refers to the terminology and underlying semantics of the evolution stages. The building or the emergence of an IE does not start from scratch and via an explicit trigger. Instead, it is established after a usually long process of seeding and cultivation of a multitude of disparate actors and supporting elements and conditions, without having a moment where such environment can be considered as ready to start from that point on. In this sense, and inspired by the terminology used in [4], a more proper term might be 'induce, seed and cultivate' instead of 'initiation \& foundation'. As mentioned, innovation-related actions can happen 'everywhere' throughout the IE's phases, and not only during the so-called 'operation' phase. The IE as a whole and its components constantly evolve. All this should be handled in such way the created catalysing environment remains sustainable. More proper terms for that might be as 'nourish \& sustain' instead of 'operation \& evolution'. Deeper changes in an IE 
can indeed happen, both due to endogenous or exogenous factors. This may impact an IE to significantly modify its general identity, profile and focus, policies, structures, etc., to adapt to the new scenario. As such, the term: 'metamorphosis' seems adequate for this stage. On the other hand, this kind of 'reset' in the IE does not happen suddenly. Instead, it is a gradual process that can take years, so processes involved in metamorphosis may start during operation, before change manifests as a stage.

'Dissolution' also happens differently in an IE. Considering the type of actors and existing infrastructures, IEs are likely to never 'disappear'. At a more daily level, individual members can come and go freely as the IE operates, some businesses and start-ups may go bankrupt, etc. At a more strategic level, an IE can lose its vitality as actors - the key ones in more particular - start disbanding the system for many possible reasons. This 'vanishing' event can be gradual and may take years, and even after that, some actors would remain in the region and could go back to be active again in any moment. Therefore, more proper terms for this stage might be as 'decommissioning \& disbanding'. Summing up, where creation, operation, evolution and metamorphosis share the same semantics in IEs as in CNs, the same thing is not true at all in the dissolution stage.

\section{Conclusions}

This research work has presented an analysis of how innovation ecosystems (IE) fit the $\mathrm{CN}$ foundations. In a previous research a bottom-up approach was applied with the aim of describing the set of processes that have been carried out when building IEs. In order to evaluate how complete the devised process-model is regarding future derivations and considering some intrinsic characteristics of IEs, ARCON was used as the reference model to be checked against in a top-down approach.

Based on literature review and on $\mathrm{CN}$ foundations, we could deduce that IEs share $\mathrm{CN}$ 's essentials and so they can be considered as another $\mathrm{CN}$ type.

After mapping our process model for building IEs against the ARCON reference model, we could verify that all ARCON aspects were present in the devised model regarding the general representation and specific modelling intent levels. Therefore, we conclude that our model is complete in terms of phases, stages of evolution as well as of endogenous and exogenous elements. This is important as the so-developed model does not intend to be a 'recipe' to build IEs. On the other hand, given a particular case, the model can be used by stakeholders as one basis to analyse, enhance or better sustain existing IEs, and be considered when conceiving future IEs. Thanks to the holistic and complete view of the whole IE building process and its life cycle, the devised model can help stakeholders to better plan and manage the time, resources allocation, and the degree of complexity of actions in different stages of IE building. This all can be helpful for predicting points of higher risks, and to prevent the whole system from achieving undesirable states.

In this research we could observe that the specific IEs model has a set of particularities not detailed by ARCON, as discussed in Section 6. IEs have a number of particularities when compared to the other fourteen types of CNs used as the basis for the ARCON conception. They are built in a decoupled and non-linear way throughout independent although inter-related phases. Processes evolve at a different and autonomous pace rather than in common cycles of global evolution. We believe that these aspects, with careful conceptual definitions (phases, processes, process \& 
activity types and instances), would benefit the users of ARCON as a reference model.

This paper is the result of an ongoing research. Next short-term steps include the development of a more formal model of the IE and its processes, comprising the mix of deliberate and emerging decoupled processes regarding the IE evolution, and the formalisation of business models at all the involved levels.

Acknowledgements. This work has been partially supported by CNPq The Brazilian Council for Research and Scientific Development funding agency.

\section{References}

1. OECD: Innovation to Strengthen Growth and Address Global and Social Challenges. URL: http://www.oecd.org/sti/45326349.pdf (2012)

2. Jackson, D.: What is an Innovation Ecosystem? National Science Foundation. 1-12 (2011)

3. Mercan, B.; Gökta, D.: Components of Innovation Ecosystems: A Cross-Country Study. International Research Journal of Finance and Economics, 76, 102-112 (2011)

4. Hwang, V. W.; Horowitt, G.: The Rainforest - The Secret to Building the Next Silicon Valley. Regenwald Publishers, USA (2012)

5. Romero, D., Molina, A.: Virtual Organisation Breeding Environments Toolkit: Reference Model, Management Framework and Instantiation Methodology. Journal of Production Planning \& Control, 21(2), 181-217 (2010)

6. Molina, A., Romero, A.: (University) Technology Parks Toolkit: Knowledge Transfer and Innovation - The Tecnológico de Monterrey Experience, 19th International ICE-Conference on Engineering, Technology and Innovation, 1-10 (2013)

7. Rabelo, R. J.; Bernus, P.: A Holistic Model of Building Innovation Ecosystems. 15th IFAC Symposium on Information Control in Manufacturing. Ottawa, Canada (2015)

8. Mercier-Laurent, E.: Innovation Ecosystems. Wiley (2011)

9. CORDIS: http://cordis.europa.eu/projects/home_en.html

10. Camarinha-Matos, L.M., Afsarmanesh, H.: Collaborative Networks: A New Scientific Discipline. Journal of Intelligent Manufacturing, 16, 439-452 (2005)

11. Camarinha-Matos, L.M., Afsarmanesh, H.: A Comprehensive Modeling Framework for Collaborative Networked Organizations. JIM, 18(5), 529-542 (2007)

12. Järvinen, P.: On Research Methods. Opinpajan kirja, Tampere, Finland (2004)

13. ISO15288: Systems and Software Engineering - System Lifecycle Processes (2008)

14. ISO15704: Industrial Automation Systems - Requirements for Enterprise Reference Architectures and Methodologies (2000; Amd 1. 2005)

15. Durst, S., Poutanen, P.: Success Factors of Innovation Ecosystems: Initial Insights from a Literature Review. CO-CREATE 2013, Aalto University, 27-38 (2013)

16. Lawlor, A.; Woodley, M.: Innovation Ecosystems. The Economist Insights. URL: www.economistinsights.com/sites/default/files/barclays_1.pdf (2014)

17. Spolidoro, R.: Innovation Habitats and Regional Development driven by the Triple Helix. IX Triple Helix International Conference, Silicon Valley, 1-23 (2011)

18. Rubens, N., Still, K., Russell, M.: A Network Analysis of Investment Firms as Resource Routers in Chinese Innovation Ecosystem. Journal of Software, 6(9), 1737-1745 (2011)

19. Carayannis, G., Barth. D., Campbell D.: The Quintuple Helix Innovation Model: Global Warming as a Challenge and Driver for Innovation. Journal of Innovation and Entrepreneurship, 1(2), 1-12 (2012)

20. Lundvall, B., Johnson B., Andersen, E.S., Dalum, B.: National Systems of Production, Innovation and Competence Building. Research Policy, 31, 213-231 (2002)

21. Afsarmanesh, H., Camarinha-Matos, L. M.: On the Classification and Management of Virtual Organization Breeding Environments. International Journal of Information Technology and Management, 8(3), 234-259 (2009) 
22. Afsarmanesh, H., Camarinha-Matos, L. M., Msanjila, S. S.: Models, Methodologies, and Tools Supporting Establishment and Management of Second-Generation VBEs. IEEE Transactions on Systems, Man and Cybernetics, 41(5), 692-710 (2011)

23. Camarinha-Matos, L. M., Ferrada, F., Oliveira, A.: Interplay of Collaborative Networks in Product Servicing. 14th IFIP Working Conference on Virtual Enterprises, 52-62 (2013)

24. Malone, T. W., Crowston, K. G., Herman, G. (Eds.): Organizing Business Knowledge: The MIT Process Handbook. Cambridge, MA: MIT Press. (2003) 